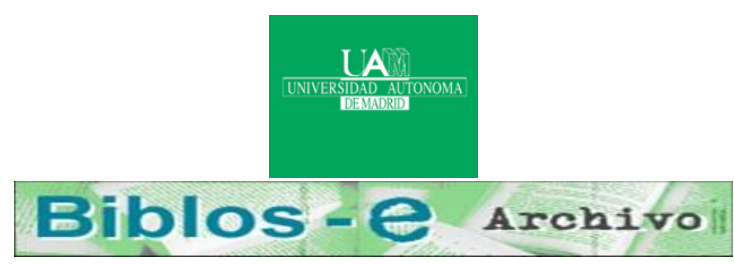

Repositorio Institucional de la Universidad Autónoma de Madrid https://repositorio.uam.es

Esta es la versión de autor de la comunicación de congreso publicada en: This is an author produced version of a paper published in:

Highlights on Practical Applications of Agents and Multi-Agent Systems: International Workshops of PAAMS. Communications in Computer and Information Science, Volumen 365. Springer, 2013. 231-239

DOI: http://dx.doi.org/10.1007/978-3-642-38061-7 23

Copyright: 2013 Springer-Verlag

El acceso a la versión del editor puede requerir la suscripción del recurso Access to the published version may require subscription 


\title{
Analysis of Gait Recognition on Constrained Scenarios with Limited Data Information
}

\author{
R. Vera-Rodriguez, S. Gabriel-Sanz, J. Fierrez, P. Tome, and J. Ortega-Garcia \\ ATVS, Escuela Politecnica Superior - Universidad Autonoma de Madrid, \\ Avda. Francisco Tomas y Valiente, 11 - 28049 Madrid, Spain \\ \{ruben.vera, silvia.gabriel, julian.fierrez, pedro.tome, javier.ortega\}@uam.es
}

\begin{abstract}
This paper is focused on the assessment of gait recognition on a constrained scenario, where limited information can be extracted from the gait image sequences. In particular we are interested in assessing the performance of gait images when only the lower part of the body is acquired by the camera and just half of a gait cycle is available (SFootBD database). Thus, various state-of-the-art feature approaches have been followed and applied to the data. Results show that good recognition performance can be achieved using such limited data information for gait biometric. A comparative analysis of the influence of the quantity of data used in the training models has been carried out obtaining results of $8.6 \%$ EER for the case of using 10 data samples to train the models, and 5.7\% of EER for the case of using 40 data for training. Also, a comparison with a standard and ideal gait database (USF database) is also carried out using similar experimental protocols. In this case 10 data samples are used for training achieving results of $3.6 \%$ EER. The comparison with a standard database shows that different feature approaches perform differently for each database, achieving best individual results with MPCA and EGEI methods for the SFootBD and the USF databases respectively.
\end{abstract}

Keywords: Biometrics, gait recognition, video surveillance

\section{Introduction}

Surveillance of public spaces is growing at an unprecedented pace in response to crime and global terrorism. For example, currently, in the UK there are reportedly more cameras per person than in any other country in the world [1]. Due to the computational improvement of the current technologies and the increase of this type of devices during these last few years in certain open areas or even closed places, the deployment of noninvasive biometric technologies becomes important for the development of automated visual surveillance systems as well as for forensic investigations. The biometric technologies more suitable for these scenarios are face and gait recognition. Others such as iris or even ear would work under more controlled conditions [5]. 
This paper is focused on gait recognition under limited data conditions. Gait is a relatively new biometric which utilizes the manner of walking to recognize an individual [8]. Compared to biometrics such as the iris or fingerprint recognition, this technique presents two main advantages: the recognition is performed at a distance and there is no need to cooperation from the users [3]. Both of these characteristics are also present in the case of face recognition, but gait has also the advantage of being able to work with low image resolution. On the other hand, the case of gait recognition is a very difficult task due to the huge amount of variability factors that can affect the gait recordings in real scenarios, such as persons walking to different directions, occlusions due to other people or clothing, different lighting conditions, etc.

In this paper six state-of-the-art feature extraction approaches for gait recognition have been followed to compare their recognition performances using a limited gait database which contains only the lower part of the body, SFootBD database (Swansea University, UK). Two configurations of this database were followed using 10 and 40 data samples for model training respectively. Then, these results were compared with a second and more ideal database used by many researchers, the USF database (University of South Florida, USA), where the gait images represent the whole body of the person. Finally, the best three individual feature approaches, GEI, EGEI and MPCA, were fused to increase the discrimination power of the systems obtaining results of $8.6 \%$ and $5.7 \%$ EER respectively for SFootBD 10 and 40 data samples in the training stage, and 3.6\% EER for USF database which uses 10 data samples for model training. This shows that even with limited gait information the results are very promising.

The remainder of the paper is organized as follows. Section 2 describes the two databases used to evaluate the performance. Section 3 describes the different feature approaches followed. Section 4 reports the experimental work and Section 5 draws the final conclusions.

\section{Gait Databases}

Due to the importance of databases which are essential tools to evaluate the biometric recognition systems, in this paper we have carried out experimental work on two gait databases whose properties differ from one another allowing to obtain a comparative analysis of the results. The first database used is the SFootBD [9]. This database is comprised of four biometric modes: footstep, gait, face and speech, using only the gait mode in this case. This database was captured without supervision obtaining therefore more realistic biometric samples (example shown in Figure 1(top)). This gait dataset is comprised of 130 users and 9893 gait image sequences but only having information for half of a gait cycle (leftright) and the lower half of the human body. The SFootBD is a much more limited database compared to the next database in terms of the amount of available information. Therefore, this database can be seen as a more realistic scenario for a gait application, e.g. a forensic case. On the other hand, the second gait 


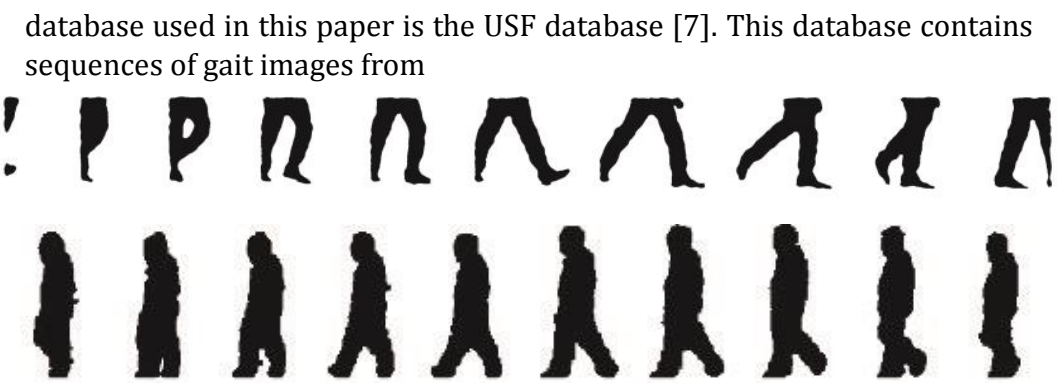

Fig.1. Examples of gait sequences of the two databases considered in this paper. SFootBD database on the top row and USF database on the bottom row.

122 users, 1870 with a half gait cycle (right-left) and the whole body shape. This database is comprised of 12 probes and 1 gallery with the persons walking under different conditions. In this paper only a subset of probe A was evaluated which is comprised of 71 users and 1458 gait sequences. This dataset contains sequences with a certain type of shoe, walking over grass. Figure 1(bottom) shows an example gait sequence from this database.

Regarding image alignment for the different feature approaches, the USF database was aligned using the position of the head. For the case of the SFootBD, the images were aligned to a central position using the position of the waist.

\section{Feature Extraction}

During the last few years, many algorithms have been developed to extract the discriminative information for gait recognition. In general, there are two main feature approaches: appearance-based and model-based [6]. Appearance-based approaches are focused on identifying persons using their silhouette, shape, geometrical measures, etc. On the other hand, modelbased approaches are focused on identifying persons using the kinematic characteristics of the walking manner. The majority of the state-of-the-art approaches are appearance-based.

In this paper, an analysis of the state-of-the-art was conducted selecting six feature approaches, which were implemented and tested with different conditions. These algorithms are: Active Energy Image (AEI) [11], Multilinear Principal Component Analysis (MPCA) [4], Gait Flow Image (GFI) [8], Gait Energy Image (GEI) [2], Motion Silhouette Contour Template (MSCT) [8] and Enhance Gait Energy Image (EGEI) [10].

Results achieved for these six feature approaches are shown in Section 4 . The three approaches obtaining best individual performance were: GEI, EGEI and MPA and are described in more detail next.

The first feature approach considered, one of the most popular to date, is called Gait Energy Image (GEI) [2]. In this case, a single image is obtained by 
averaging the binary silhouettes of a pedestrian over one gait cycle. Therefore, this method is an appearance-based approach. As Figure 2 shows, the image obtained represents by means of the intensity of each pixel, the frequency (energy) of body occurrence at the position of each pixel for a complete walking cycle. This algorithm was developed in 2006 [2] and although it is not as new as the other ones, the good results reflect the effectiveness of it. This method allows an easy implementation and reduces the time, storage and computational costs but it is heavily affected by factors such as the clothing and persons carrying objects.

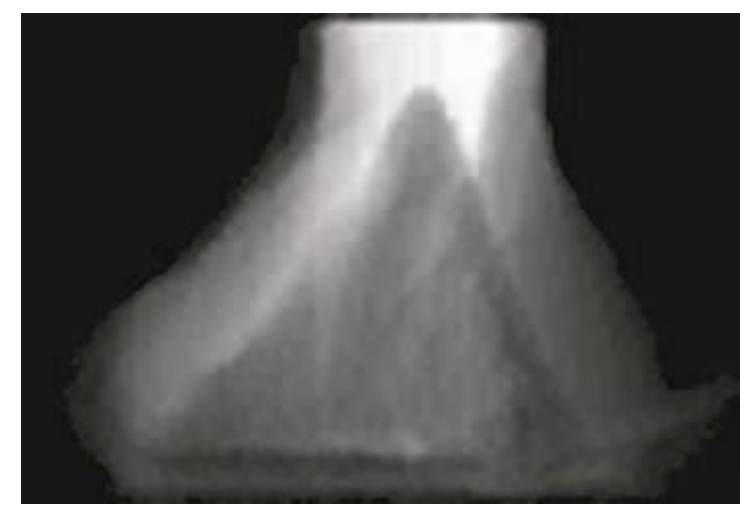

Fig.2. Example of Gait Energy Image (GEI) for SFootBD database.

The second feature approach considered, called Enhanced Gait Energy Image (EGEI) [10], is based on enhancing the previous GEI method. For this, an averaged GEI image representing each user class is used to construct a dynamic weight mask (DWM) by variance analysis. This mask is applied to the original GEI images to obtain the EGEI images. Finally, this method uses a Gabor filter bank in order to emphasize the most discriminative parts of the body image as shown in Figure 3. This technique is computationally more expensive than the GEI method, but allows to improve the results in cases of having much noisier environments.

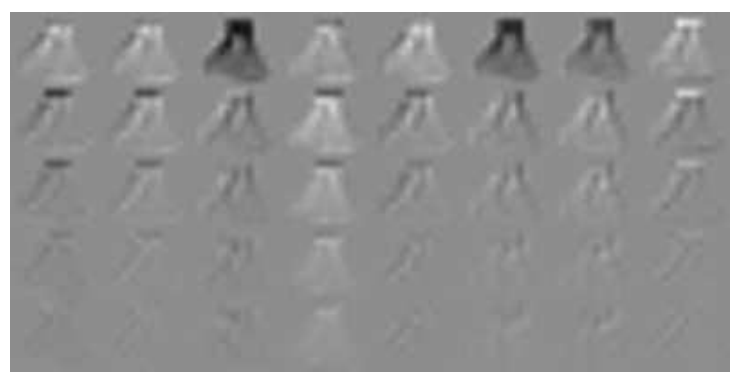


Fig.3. Example of Enhanced Gait Energy Image (EGEI) for SFootBD database.

The third approach considered in this paper, called Multilinear Principal Component Analysis (MPCA) [4], is an extension of the popular algorithm PCA. As can be seen in Figure 4, the data is arranged in several dimensions to form a tensor. In our case, four dimension tensors are used: two spatial dimensions of the images, a time dimension and another dimension for the different data examples. Once the tensor is ready, MPCA can drastically reduce the high dimensionality of the original data into low dimension feature vectors which are used in the classification stage.

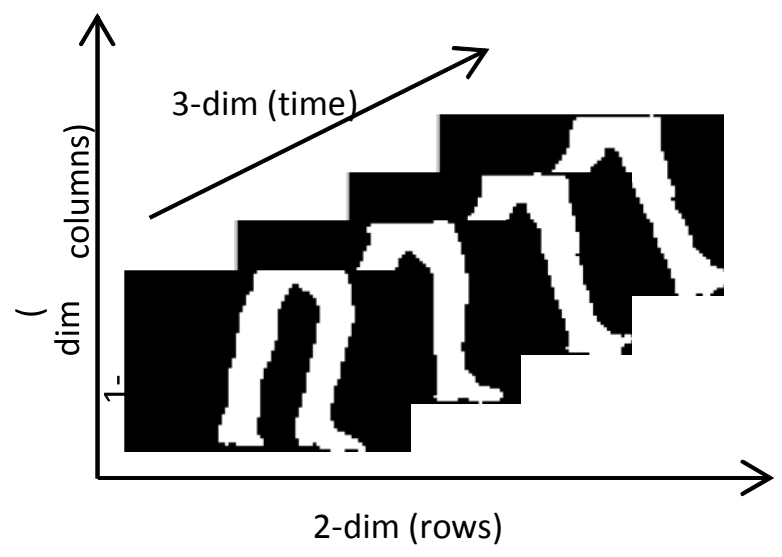

Fig.4. Example of Multilinear Principal Component Analysis (MPCA).

\section{Experimental Work}

\subsection{Experimental Protocol}

To carry out the experimental work to study the discriminative power of different gait recognition systems using limited data, both databases considered were divided into training and test sets. Two configurations were considered for SFootBD database, using 10 and 40 data samples for model training, being 59 and 40 the number of users present in the training set respectively. The case of USF database was only set to 10 data samples for model training, having 71 users. It is worth mentioning that SFootBD is comprised of 130 users which are all present in the test set, so in this configuration there is a impostor dataset comprising data for the remaining users, which makes this an open-set scenario, more challenging than the case of the USF database.

Reduction of feature dimensionality was performed over the six feature approaches considered. Firstly, principal component analysis (PCA) was 
applied, analysing different number of principal components (PC). The case of MPCA did not require to use PCA. Later, linear discriminant analysis (LDA) was used to further extract the most relevant information. Finally, support vector machine (SVM) with a RBF kernel was employed as the classifier to obtain the recognition results. Experiments are carried out for both identification ( 1 vs. all) and verification (1 vs. 1 ) working modes. In the first case, top rank identification performance is obtained using cumulative match characteristic (CMC) curves, and for the case of verification DET curves are obtaining giving the equal error rate (EER) as a measure of the performance.

\subsection{Evaluation of Results}

The first experiment was set to compare the individual performance of the six feature approaches considered (AEI, MSCT, GFI, GEI, EGEI and MPCA) over the two gait databases using the same data configuration, i.e., 10 data samples for training. Results are shown in Table 1. It is interesting to note that the number of PCA components was smaller in all cases but one (MPCA) for the SFootBD compared to the USF database, most likely for the lower amount of information contained (approximately a quarter of the information as only half of the gait cycle is visible for the lower part of the body).

\begin{tabular}{|c|c|c|c|c|c|c|}
\hline \multirow{2}{*}{} & \multicolumn{2}{|c|}{ SFOOTBD 10 } & \multicolumn{2}{c|}{ SFOOTBD 40 } & \multicolumn{2}{c|}{ USF 10 } \\
\cline { 2 - 7 } & Rank 5 ID & EER & Rank 5 ID & EER & Rank 5 ID & EER \\
\hline AEI & 69.1 & 16.4 & 82.5 & 11.4 & 85.6 & 9.2 \\
\hline MSCT & 75.1 & 13.5 & 85.8 & 9.4 & 88.3 & 7.9 \\
\hline GFI & 72.6 & 14.4 & 85.2 & 9.8 & 86.0 & 9.6 \\
\hline GEI & 77.8 & 12.7 & 86.5 & 8.6 & 96.4 & 4.0 \\
\hline EGEI & 79.8 & 11.9 & 88.0 & 7.6 & 96.4 & 4.1 \\
\hline MPCA & 83.2 & 9.8 & 89.9 & 6.4 & 95.5 & 5.4 \\
\hline FUSION & $\mathbf{8 5 . 6}$ & $\mathbf{8 . 6}$ & $\mathbf{9 0 . 5}$ & $\mathbf{5 . 7}$ & $\mathbf{9 7 . 1}$ & $\mathbf{3 . 6}$ \\
\hline
\end{tabular}

Table 1. Results achieved for both SFootBD database with 10 and 40 signals to train the models and USF database, with rank 5 identification rate and EER both in \%.

As can be seen in Table 1, the GEI, EGEI and MPCA approaches obtained much better individual performance compared to AEI, MSCT, GFI, for both identification (rank 5) and verification (EER) experiments. Much better results were achieved for the case of USF database as was expected. But results in the order of $10 \%$ EER were achieved for the SFootBD which is 
good. It is interesting to note that the different feature approaches perform differently from one database to another. GEI and EGEI approaches perform better than MPCA for the USF database, while MPCA is the best approach for the SFootBD. This is due to the missing information of the SFootBD, as GEI and EGEI approaches are proven to work very well when the whole silhouette is visible.

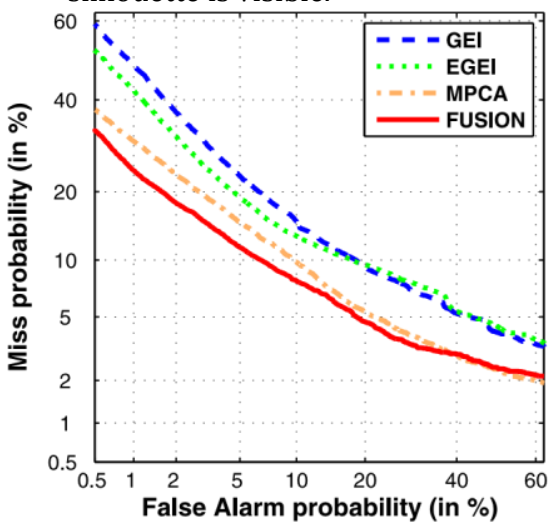

(a) SFootBD 10

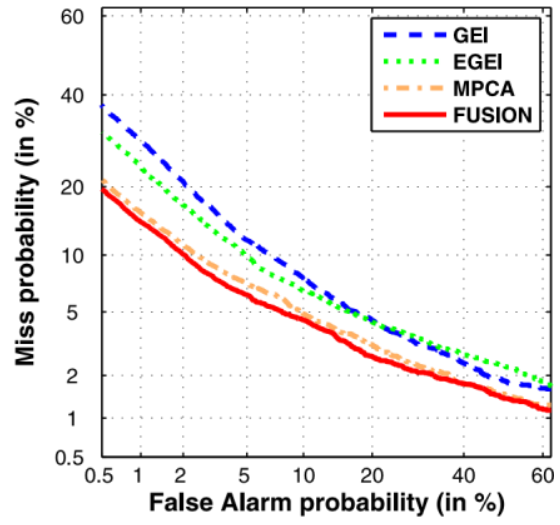

(b) SFootBD 40

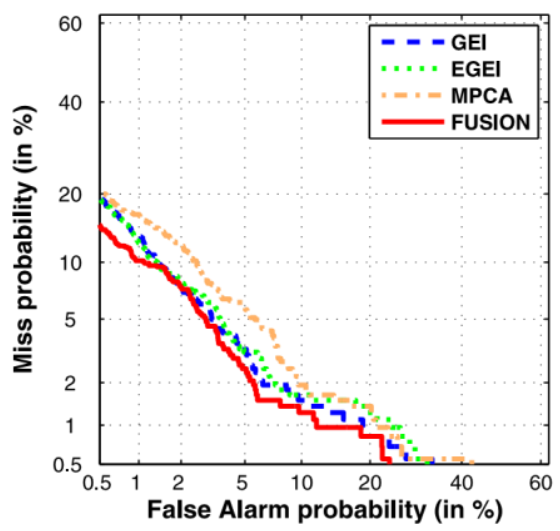

(c) USF DB 10

Fig.5. DET Curves for both SFootBD database with 10 and 40 signals to train the models and USF database comparing the three best feature approaches and their fusion at the score-level.

The second experiment was the analysis of the influence of the amount of training data in the performance. For this only SFootBD was used with the two configurations of 10 and 40 data samples for model training. Table 1 shows how the results improve significantly when more data is included in the training models, getting close to the results achieved for the USF database. The same trends in the approaches are observed. The third 
experiment was the fusion of the best three feature approaches in order to further improve the recognition performance of the system for a limited gait database. The fusion was carried out at the score-level using a simple product rule.

Figure 5 shows four DET curves for the three selected feature approaches and the fusion for the SFootBD (configurations 10 and 40) and USF databases respectively. As can see in Figures 5(a) and 5(b), the approach which provides better results for the case of the SFootBD is MPCA ( $9.8 \%$ and $6.4 \%$ EER respectively). The fusion of the three approaches achieved also a small improvement in terms of identification rate $(85.6 \%$ and $90.5 \%$ for rank 5 respectively) and EER ( $8.6 \%$ and $5.7 \%$ respectively). Table 1 shows an overview of the main results achieved. In contrast to these results Figure 5(c) shows results for the case of the USF database where the three feature approaches provide very similar identification rates, achieving a slightly better performance for EGEI ( $96.4 \%$ of rank 5 identification rate). The fusion of the three approaches achieves a small improvement obtaining $97.1 \%$ rank 5 identification rate and $3.6 \%$ EER.

\section{Conclusions}

In this paper, an evaluation of gait recognition systems over data with limited information (SFootBD) has been carried out. For this, six stateof-the-art feature approaches (AEI, MSCT, GFI, GEI, EGEI and MPCA) have been applied to the gait data. Similar experimental work has been followed over an ideal gait database (USF database) in order to compare results. In both cases best individual performance has been achieved for GEI, EGEI and MPCA feature approaches and a fusion of the three has been carried out at the score-level. As expected, there are significant differences in the performance of these approaches over the two databases, but more than acceptable results (85.6\% of rank 5 identification rate and $8.6 \%$ of EER) have been achieved over the limited gait database, showing that using only the lower part of the body provides significant discriminative information for person recognition.

\section{Acknowledgements}

This work has been supported by projects Contexts (S2009/TIC-1485), BioChallenge (TEC2009-11186), Bio-Shield (TEC2012-34881) and "Catedra UAM-Telefonica". Ruben Vera-Rodriguez is supported by a Juan de la Cierva Fellowship from the Spanish MINECO.

\section{References}

1. http://www.guardian.co.uk/uk/2009/mar/02/westminstercctvsystem-privacy (2009) 
2. Han, J., Bhanu, B.: Individual recognition using gait energy image.IEEE Trans. Pattern Anal. Mach. Intell. 28(2), 316-322 (2006)

3. Han, S., Zhi-Wu, L., Guo-Yue, C.: A gait recognition method usingL1-PCA and LDA. In: Proc. International Conference on Machine Learning and Cybernetics, vol. 6, pp. 3198 - 3203 (2009)

4. Lu, H., Plataniotis, K.N., Venetsanopoulos, A.N.: Multilinear principal component analysis of tensor objects for recognition. In: Proc. of the 18th International Conference on Pattern Recognition, vol. 2, pp. 776779 (2006)

5. Nixon, M., Bouchrika, I., Arbab-Zavar, B., Carter, J.: On the use ofbiometrics in forensics: gait and ear. In: Proc. of European Signal Processing Conference (EUSIPCO) (2010)

6. Nixon, M.S., Carter, J.N.: Automatic recognition by gait. Proceedings of the IEEE 94(11), 2013-2024 (2006)

7. Sarkar, S., Phillips, P.J., Liu, Z., Vega, I.R., Grother, P., Bowyer,K.W.: The humanID gait challenge problem: data sets, performance, and analysis. IEEE Transactions on Pattern Analysis and Machine Intelligence 27(2), 162-177 (2005)

8. Trivino, G., Alvarez-Alvarez, A., Bailador, G.: Application of thecomputational theory of perceptions to human gait pattern recognition. Pattern Recognition 43(7), 2572-2581 (2010)

9. Vera-Rodriguez, R., Mason, J., Fierrez, J., Ortega-Garcia, J.: Comparative analysis and fusion of spatio-temporal information for footstep recognition. IEEE Transactions on Pattern Analysis and Machine Intelligence 99 (2012)

10. Yang, X., Zhou, Y., Zhang, T., Shu, G., Yang, J.: Gait recognitionbased on dynamic region analysis. Signal Processing 88(9), 2350- 2356 (2008)

11. Zhang, E., Zhao, Y., Xiong, W.: Active energy image plus 2DLPPfor gait recognition. Signal Processing 90(7), 2295-2302 (2010) 